\title{
Air-coupled Ultrasound Wave Propagation in Glued Laminated Timber Structures Applied to Bonding Quality Assessment
}

\author{
S. J. Sanabria ${ }^{1}$, R. Furrer ${ }^{1}$, J. Neuenschwander ${ }^{1}$, P. Niemz ${ }^{2}$, U. Sennhauser ${ }^{1}$ \\ ${ }^{1}$ Empa, Swiss Federal Laboratories for Materials Science and Technology, \\ Electronics / Metrology / Reliability Laboratory, CH-8600 Dübendorf, Switzerland \\ ${ }^{2}$ ETH Zürich, Wood Physics and Non-Destructive Testing Methods Laboratory, CH-8093 Zürich, Switzerland
}

\begin{abstract}
Non-destructive assessment of glulam laminations is necessary in order to prevent security hazards. Air-coupled ultrasound $(\mathrm{ACU})$ is sensitive to delamination, reproducible and allows for high scanning resolution. In this work we developed a novel ACU imaging system in normal transmission for inspection of up to $280 \mathrm{~mm}$ high glulam. The coupling and propagation of ACU waves in glulam were investigated with plane wave theory applied to an orthotropic model of wood as a function of grain and ring angles. Finite Difference Time Domain (FDTD) simulations were also carried out. The systematic amplitude heterogeneity in defect-free regions was linked to energy flux shifts and mode conversion phenomena, and compensated for in the images. Specific amplitude tracking and difference imaging algorithms allowed successful imaging of bonding and saw cut defects.
\end{abstract}

\section{INTRODUCTION}

$\mathrm{W}$ ORLDWIDE there exists 3.9 bn ha forest which absorb pollution and use solar energy to generate a total of $384 \mathrm{bn} \mathrm{m}^{3}$ renewable biomass, out of which $0.4 \mathrm{bn} \mathrm{m}^{3}$ of sawn wood is annually produced [1]. Glued laminated timber (glulam) is manufactured by lengthways sawing timber lamellas (typically 30 to $40 \mathrm{~mm}$ thick and $200 \mathrm{~mm}$ wide [2]), which are then dried, planed, end-jointed, glued and stacked. This arrangement disperses or sorts out natural defects present in wood, allowing for large structural members (more than $2 \mathrm{~m}$ high and $50 \mathrm{~m}$ long) of straight or curved shapes [3]. The integrity and load bearing capacity of the glue lines need to be periodically tested, in order to detect in service cracking and delaminations. Current European standardized methods rely on visual assessment [4] and destructive tests on random samples from the total production [5]. Infrared thermography detects changes in thermal conductivity in the presence of delamination [6]; however, it is limited to thin veneer inspection. X-ray and neutron computed tomography can segment the presence or absence of adhesive in wood composites $[7,8]$; however, their hazardous nature constrains their use on site. Ultrasonics are highly sensitivity to

This work was supported by the Swiss National Science Foundation under contract 200021-115920. delaminated interfaces, and portable to the construction. Traditional experimental glulam diagnostics were performed with contact transducers $[9,10]$. The drawbacks are poor, coupling pressure dependent amplitude reproducibility, penetration of coupling agents into the sample and difficulties of performing imaging with sufficient spatial resolution. Aircoupled ultrasound (ACU) is highly reproducible (amplitude error below 1\% [11]) and allow for high resolution scanning. However, only $0.3 \%$ of input energy is coupled into wood samples, due to the quasi-specular reflection in interfaces air/solid. This has so far limited the inspection to veneer [12], solid wood [13] or two $5 \mathrm{~mm}$ thick boards glued together [11]. An ultrasonic wave propagation model in glulam is neither available. [14] investigated travel time tomography in standing trees, with some corrections to account for ellipsoidal anisotropy. [15] analyzed anisotropic coupling of ultrasound energy in the fiber direction of waterlogged pine boards.

In this work we investigate the coupling and propagation of ACU waves in glulam. First an ACU imaging system was developed for acquisition of ultrasound signals with sufficient signal-to-noise ratio. Secondly, analytical and numerical modeling of wave paths was performed. Finally, bonding defect assessment was demonstrated.

\section{ACU GLULAM INSPECTION SYSTEM}

A pulsed ACU normal transmission imaging system was developed (Fig. 1). Two ACU transducers, namely transmitter TX and receiver RX, were separated at least $80 \mathrm{~mm}$ from the sample and scanned in the bonding plane $\mathrm{XY}$ with a pixel size of $1 \times 5 \mathrm{~mm}^{2}$ by a computer-controlled 3 axis mechanical scanner (ISEL Germany AG, Eichenzell). The insonification axis $\mathrm{Z}$ was perpendicular to the lamination plane. The transducers were $120 \mathrm{kHz}$ broadband planar Gas Matrix Piezoelectric Composites (NCG100-D50, The Ultran Group Inc., State College, PA). For each scanned position a pulsed excitation was generated by an arbitrary waveform generator (NI PCI-5421, National Instruments Corp., Austin, TX, USA) and amplified to $600 \mathrm{Vpp}$ with a gated unit (RPR4000, RITEC Inc., Warwick, RI, USA), which output was then driven into the TX. A shunt $50 \Omega$ load at the output of the RPR4000 was 


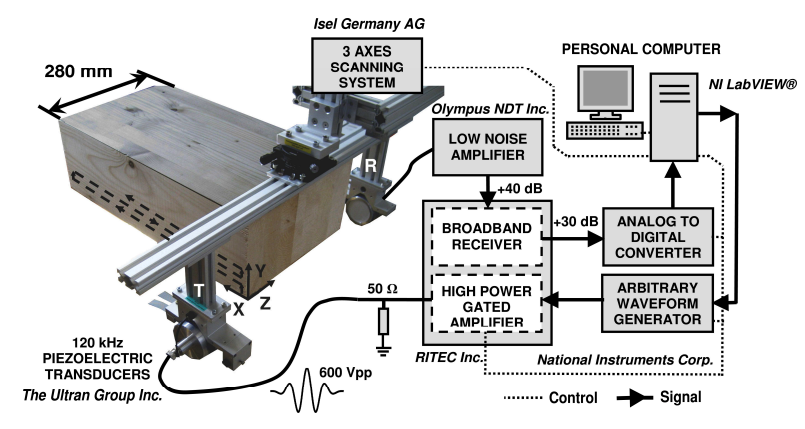

Fig. 1. ACU glulam inspection setup

used for broadband impedance matching with the TX. The received ultrasonic signals were amplified $40 \mathrm{~dB}$ with a lownoise unit (5660C, Olympus NDT Inc., Waltham, MA, USA) and then filtered and conditioned by a broadband receiver (RPR4000). The waveforms were sampled with $2.5 \mathrm{MS} / \mathrm{s}$ and 14 bits digital resolution (NI PCI-5122).

Experiments were performed in $65 \mathrm{~mm}$ thick and $280 \mathrm{~mm}$ thick 7 lamellas glulam made from Norway spruce (Picea abies Karst.). In the latter we bridged $115 \mathrm{~dB}$ transmission losses in defect-free material with a signal-to-noise (SNR) ratio of $20 \mathrm{~dB}$. Since the scanning step was smaller than the minimum lateral resolution (given by the wavelength, about $10 \mathrm{~mm}$ ), overlapped averaging of spatially adjacent waveforms which increased the SNR up to $40 \mathrm{~dB}$.

\section{WAVE PROPAGATION MODEL}

\section{A. Analytical model}

The ACU NTM wave propagation in glulam was modeled with plane wave theory in aelotropic crystals [16] and an orthotropic model of wood elasticity [17]. Table 1 summarizes typical spruce stiffness values [18], the density is $\rho=494 \mathrm{~kg} \mathrm{~m}^{-3}$. Assuming homogeneous anisotropy along the wave path within a single lamella, the wave normal $\mathbf{n}$ is parallel to $\mathrm{Z}$. We computed phase velocities $c$ and polarization vectors $\mathbf{p}$ for each timber lamella by using eigendecomposition of the Christoffel tensor $\Gamma_{i j}$ and by performing vector transformation between laminate $\mathrm{XYZ}$ ( $\mathbf{n}$, $\mathbf{p}$ ) and material bases LRT ( $\left.\mathbf{n}^{\prime}, \mathbf{p}^{\prime}\right)$, where $\mathrm{L}$ is the growth or fiber direction, and $\mathrm{R}$ and $\mathrm{T}$ are radial and tangential to the annual rings, respectively (Fig. 2a).

$$
\begin{aligned}
& \sum_{k=1}^{3}\left(\Gamma_{i k}-\rho c^{2} \delta_{i k}\right) p_{k}{ }^{\prime}=0 \quad \Gamma_{i k}=\sum_{j=1}^{3} \sum_{l=1}^{3} C_{i j k l} n_{j}{ }^{\prime} n_{l}{ }^{\prime} \\
& i=1 \ldots 3 \quad \mathbf{n}^{\prime}=\mathbf{A}^{\mathbf{T}} \mathbf{n} \quad \mathbf{p}=\mathbf{A p}^{\prime} \\
& \mathbf{A}=\left(\begin{array}{ccc}
\cos \theta & -\sin \phi \sin \theta & 0 \\
0 & \cos \phi & -\sin \phi \\
-\sin \theta & -\sin \phi \cos \theta & \cos \theta \cos \phi
\end{array}\right)
\end{aligned}
$$

The vector transformation was locally defined for each lamella as a function of ring angle $\phi$ and grain angle $\theta$

(Fig. 2b). According to the glulam manufacture procedure (I.) $\mathrm{L}$ is well-aligned with $\mathrm{Z}$, thus $\theta=0^{\circ}$ and plane wavefronts
TABLE I

\begin{tabular}{llllll}
\multicolumn{7}{c}{ ELASTIC CONSTANTS FOR NORWAY SPRUCE (GPa) } \\
$\mathrm{C}_{\text {LLLL }}$ & 16.60 & $\mathrm{C}_{\text {LLRR }}$ & 0.44 & $\mathrm{C}_{\text {LRLR }}$ & 0.04 \\
$\mathrm{C}_{\text {RRRR }}$ & 0.79 & $\mathrm{C}_{\text {LLTT }}$ & 0.32 & $\mathrm{C}_{\text {LTLT }}$ & 0.78 \\
$\mathrm{C}_{\text {TTtT }}$ & 0.45 & $\mathrm{C}_{\text {RRTT }}$ & 0.20 & $\mathrm{C}_{\text {RTRT }}$ & 0.63
\end{tabular}

are perpendicular to the RT plane. Fig. 2a shows polar plots of $c$ and $\mathbf{p}$ as a function of $\phi$. Three modes propagate through timber, namely QP (quasi longitudinal), QSV (quasi shear, in-plane) and SH (purely shear, out of plane). Fig. 2b illustrates ACU NTM wave propagation through a single timber lamella. Due to the non-sphericity of the velocity surface, the energy flux $\xi$ deviates for $\mathbf{n}$, which results in a lateral shift $\chi^{\left({ }^{\circ}\right)}$ from $\mathrm{Z}$ :

$\chi=\angle(\mathbf{n}, \xi) \quad \xi_{i}{ }^{\prime} \propto C_{i j k l} p_{j}{ }^{\prime} p_{k}{ }^{\prime} n^{\prime} \quad \xi=\mathbf{A} \xi^{\prime}$

Therefore, only a portion of the transmitted sound field is captured by the ACU NTM receiver, large $\chi$ result in strong amplitude losses. The shifts are highly dependent on the coupled mode and $\phi$ (Fig. 2c). In the principal material axes $(\mathrm{R}$ and $\mathrm{T}$ ) all modes propagate aligned with $\mathbf{n}$. The SH mode show the smallest shifts $\left(<6^{\circ}\right)$. The QP mode tends to align with $\mathrm{T}$ and $\mathrm{R}$ with an equilibrium point at $\phi=30^{\circ}$ (pure $\mathrm{P}$ mode) and a $30^{\circ}$ shift towards $\mathrm{R}$ at $\phi=50^{\circ}$; deviations from $\mathrm{T}$ introduce smaller $\chi\left(<10^{\circ}\right)$. The QSV mode typically shows largest $\chi$ (up to $60^{\circ}$ ) with a sign opposite to QP. A wave surface cusp at $\phi=39^{\circ}$ aligns $\xi$ and $\mathbf{n}$. The shifts were found to be highly reproducible (error $<0.9^{\circ}$ for $\mathrm{QP}$ ) for stiffness data from different authors. We as well analyzed the effect of small grain misalignments $\theta \neq 0^{\circ}$. Due to the large anisotropy ratio between RT and $\mathrm{L}\left(c_{L}=5000 \mathrm{~m} \mathrm{~s}^{-1}\right)$, $\theta=0.1^{\circ}$ introduces for QP $2.5^{\circ}$ out-of plane shifts towards $\mathrm{L}$ Moreover, large $\theta \neq 0^{\circ}$ couple QSH modes, leading to

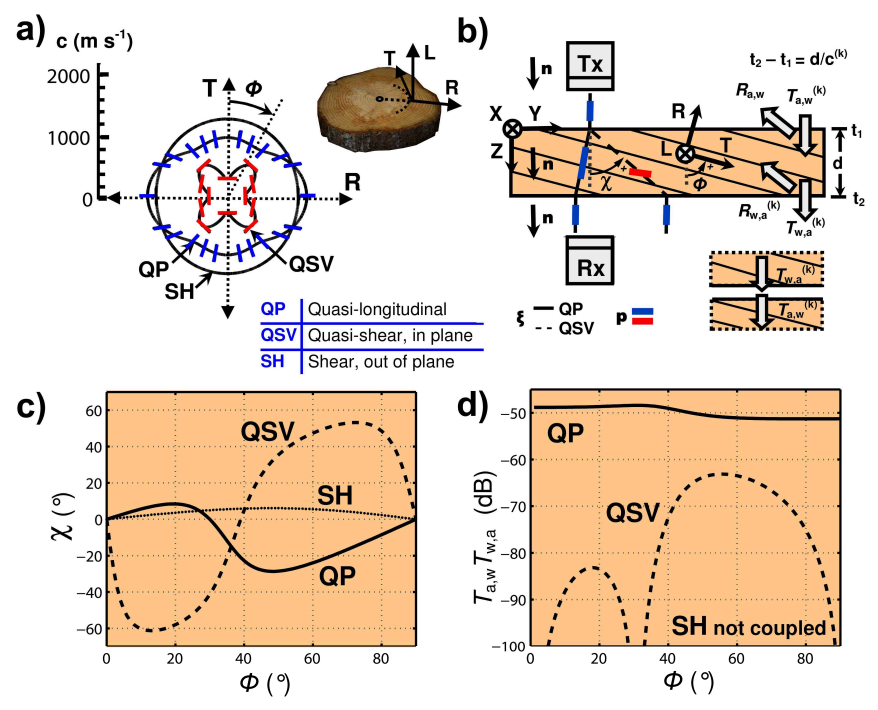

Fig. 2 Wave propagation model. a) Velocity surface for Norway spruce (Picea abies Karst.) in the RT plane. b) Wave parameter definition. c) Angular deviation of energy flux from wave normal as function of the ring angle. c) Coupling losses of wave modes as a function of the ring angle. 
birefringence phenomena. Experimental observations suggest that the beam spread in $\mathrm{L}$ and cancellation of random shifts minimize the effect of grain shifts in glulam inspection.

The wave propagation in $N$ layers glulam was modeled as a $2 N+1$ layers system ( $N$ lamellas, $N-1$ glue lines, 2 air gaps). In the air-solid interfaces mode conversion between $P$ waves (air) and QP and QSV waves in timber occur, which leads to beam splitting (Fig. 2b) due to the different $\xi$ of each mode; the same occurs in solid-solid interfaces. The $\mathrm{SH}$ mode is not coupled for $\theta=0^{\circ}$ since $\mathbf{p} \perp \mathbf{n}$. Therefore, a total of different $2^{2 N-1}$ wave paths were possible in the composite. Secondary wave reflections between timber lamellas could be neglected at $120 \mathrm{kHz}$ due to the $230 \mathrm{~dB} \mathrm{~m}^{-1}$ material attenuation coefficient in wood (measured in [19]). The 2layer transmission $T^{M}$ and reflection coefficients $R^{Q}$ and reflection coefficients for specific incident $I$, transmitted $M$ and reflected $Q$ modes (QP or QSV) were computed from traction and displacement continuity conditions [16]:

$$
\begin{cases}p_{k}{ }^{I}=-\sum_{M=1}^{3} R^{M} p_{k}{ }^{M}+\sum_{N=1}^{3} T^{N} p_{k}{ }^{N} & k=1 \ldots 3 \\ \rho^{I} c^{I} p_{i}{ }^{I}=\sum_{M=1}^{3} R^{M} \rho^{M} c^{M} p_{i}{ }^{M}+\sum_{N=1}^{3} T^{N} \rho^{N} c^{N} p_{i}{ }^{N} & i=1 \ldots 3\end{cases}
$$

The three layers transmission coefficient $T_{a, w} T_{w, a}$ for an air/wood/air system are plotted in Fig. 2d, and correspond to the ACU NTM coupling losses for specific modes. They are also equivalent to the maximum amplitude reduction observed when transmitting through a delaminated interface with respect to defect-free glulam. In practice, the amplitude contrast in delaminated interfaces decreases due to interference phenomena in the glue line; for an air gap of $10 \mu \mathrm{m}$ the amplitude contrast of the QP mode is reduced from 50 to $20 \mathrm{~dB}[11,19]$. The QSV mode is typically coupled 20 to $30 \mathrm{~dB}$ below the QP mode, at ring angles between $45^{\circ}$ and $70^{\circ}$ the transmission difference is reduced to $15 \mathrm{~dB}$.

\section{B. Numerical simulation}

The analytical model was validated with $2.5 \mathrm{D}$ Finite Difference Time Domain simulations (FDTD). We used a rotated staggered grid (RSG), which was first described in cracked material research [20], in order to accurately discretize the air/wood discontinuities (voxel size: $200 \mu \mathrm{m}$, time step: $100 \mathrm{~ns}$ ). The transducer surface was decomposed in Huygens wavelets, which reduced the air path discretization to the vicinity of the sample surfaces. The stiffness tensor was

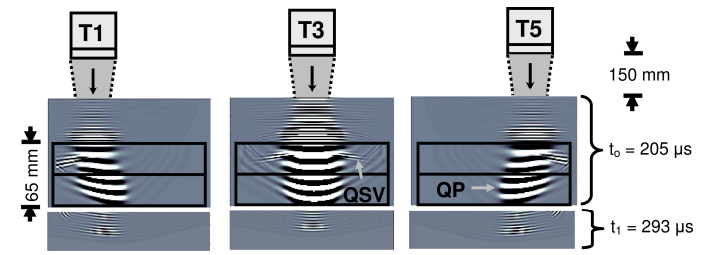

Fig. 3. FDTD time snapshots corresponding to transmitter positions T1, T3 and T5 from Fig. 2 (top). Mode conversion, energy flux shift deviations and ultrasound beam diffraction are observed.

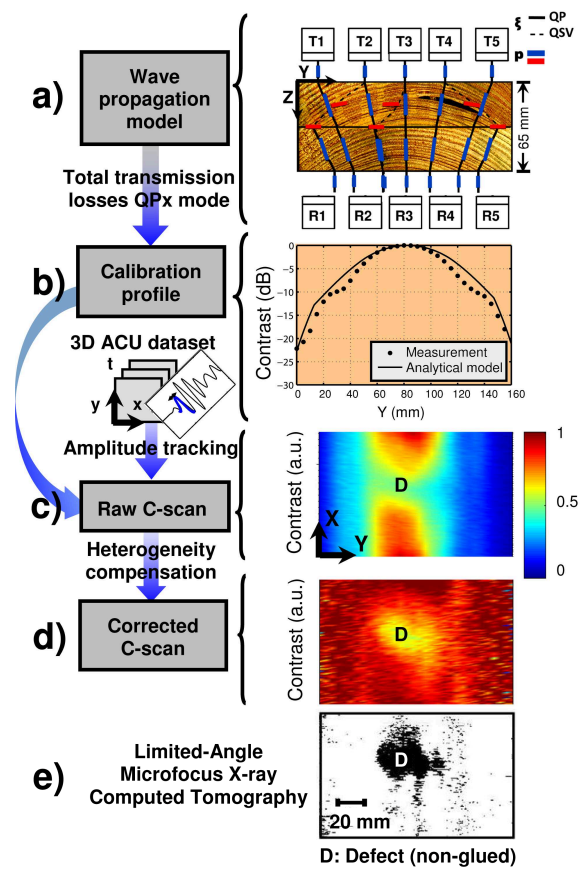

Fig. 4 Wave propagation and bonding defect imaging in two layers glulam.

locally computed by fitting the year ring structure to a cylindrical orthotropy model. The numerical model incorporated $\xi$ shifts, mode conversion and beam diffraction (Fig. 3). The beam spread is largest in L; a circular Gaussian beam transmitted through $65 \mathrm{~mm}$ glulam was transformed into a Gaussian ellipsoid such that $\sigma_{L} / \sigma_{R T}=3.3$.

\section{RESULTS AND DISCUSSION}

\section{A. Two lamella systems}

Fig. 4a shows wave paths for a $65 \mathrm{~mm}$ thick two lamella system. The NTM transmission profile was accurately predicted in Fig. $4 \mathrm{~b}$ by computing coupling and beam shift losses, the latter calculated by shifting a position-independent sound field with the computed shifts and calculating the overlap region with the receiver transducer. The unexplained amplitude variability was reduced from 25 to $3 \mathrm{~dB}$. Fig. $4 \mathrm{c}$ shows an amplitude image obtained from the ultrasound dataset with a tracking algorithm, which exploited the spatial continuity of ACU datasets to follow the amplitude of the first incoming oscillation [8], the latter corresponding to the fastest (denoted as QPx) mode. The amplitude variability pattern masks the defect position (non-glued area of $30 \times 60 \mathrm{~mm}^{2}$ ). As expected, the variability pattern is determined only by $\phi$ and, therefore, it is repeatable in Z. Normalization with Fig. $4 \mathrm{~b}$ compensates the heterogeneity pattern highlighting the defect position (Fig. 4d). The results were validated with a novel limited-angle Microfocus X-ray Computed Tomography method [8] (Fig. 4e). The position and geometry of the defect detected with both techniques is consistent. In practice, the normalization profile may be obtained from a calibration measurement for defect-free material. Fig. 3 shows selected FDTD snaps for the same setup. The beam shift effect is observed as expected for T1 and T5. QSV mode coupling can 
as well be assessed, even though for this setup the QPx mode is typically at least $20 \mathrm{~dB}$ larger. The transmitted wavefronts are approximately aligned with the insonification axis $Z$. The color scale has been adjusted to visualize input and output waves on the same plot.

\section{B. Seven lamella systems}

Fig. 5a shows typical wave paths for 7 lamellas glulam. A total of 8192 mode combinations are possible. The QPx mode is only imaged at center beam width. It is followed by secondary paths, including a complex interference pattern of QP/QSV modes with small total shifts, and edge reflections E, which are coupled at all sample width positions. They show less effective lateral resolution than the QPx mode, due to the larger uncertainty region defined by the accumulation of beam trajectories of the modes interfering at the receiver. Fig. $5 \mathrm{~b}$ shows bonding imaging results in a glulam beam before and introducing a saw cut defect. An amplitude decrease could be identified in the defect area, which was partially masked by the variability pattern in defect free regions. Defects larger than $100 \times 100 \mathrm{~mm}^{2}$ could be successfully imaged A difference imaging procedure (Fig. 5c) was used to compare the two images [21]. The amplitude variability was reduced from 15 to $5.5 \mathrm{~dB}$, which allowed for accurate segmentation of the defect geometry using MAP binarization procedure [8].

\section{CONCLUSIONS AND OUTLOOK}

We demonstrated successful ACU flaw imaging up to $280 \mathrm{~mm}$ high glulam. The signal processing strategies profited from the high ACU spatial resolution (amplitude tracking, overlapped averaging) and reproducibility (difference imaging). The systematic variability patterns in defect-free regions were linked to anisotropic energy flux shifts. For 2 lamellas systems the QPx mode is dominant; the shifts introduce $25 \mathrm{~dB}$ amplitude variability, which could be modeled down to $3 \mathrm{~dB}$. For 7 lamellas system a complex $\mathrm{QP} / \mathrm{QSV}$ interference pattern and edge reflections were

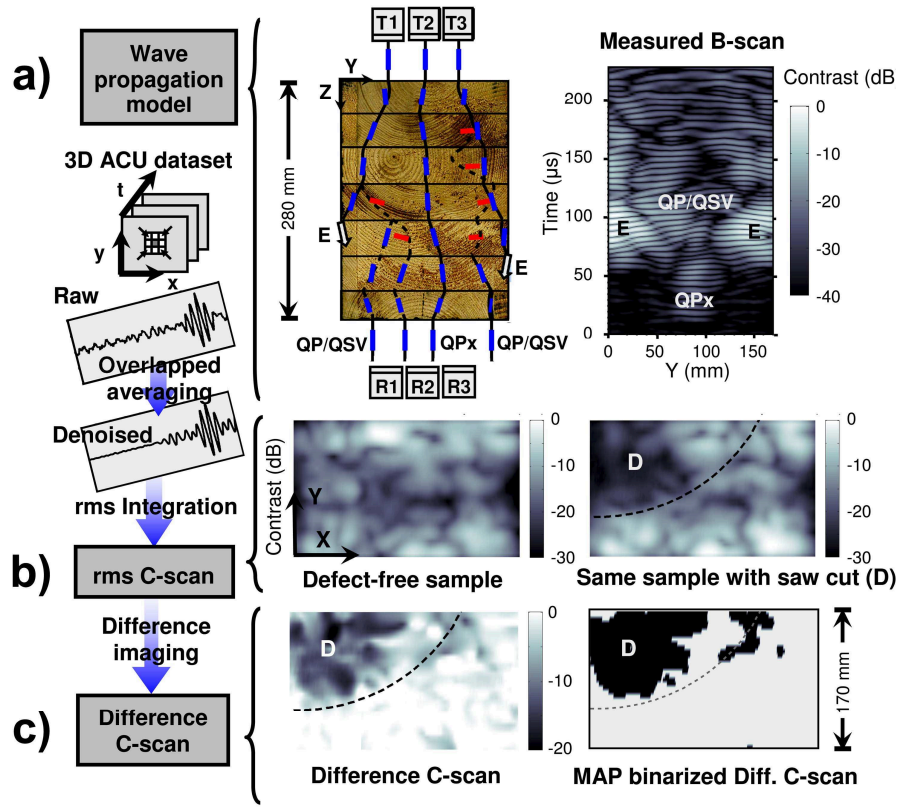

identified. Work is planned for defect detection in glulam higher than $500 \mathrm{~mm}$. Wave path inversion based on the described models will also be investigated.

\section{ACKNOWLEDGMENT}

The authors acknowledge the work of O. Tolar, L. Zumofen, F. Binkert, D. Gómes, C. Weiss and D. Mächler in the development of the ACU system.

\section{REFERENCES}

[1] "State of the World's Forests," Food and Agriculture Organization of the United Nations, Rome 2009.

[2] EN 386, "Glued laminated timber - Performance requirements and minimum production requirements.," 2001.

[3] J. Bodig and B. A. Jayne, Mechanics of Wood and Wood Composites. Scarborough, Ontario: Van Nostrand Reinhold Publishing, 1982.

[4] EN 391, "Glued laminated timber: Delamination test of glue lines," 2001.

[5] EN 14080, "Timber structures - Glued laminated timber Requirements," 2005.

[6] H. Berglind and A. Dillenz, "Detection of glue deficiency in laminated wood with pulse thermography," Journal of Wood Science, vol. 49, pp. 216-220, 2003.

[7] K. Osterloh, C. Raedel, U. Zscherpel, D. Meinel, U. Ewert, T. Buecherl, and A. Hasenstab, "Fast neutron radiography and tomography of wood," Insight, vol. 50, pp. 307-311, Jun 2008.

[8] S. J. Sanabria, P. Wyss, J. Neuenschwander, P. Niemz, and U. Sennhauser, "Assessment of Glued Timber Integrity by Limited-angle Microfocus X-ray Computed Tomography," Submitted to Holz RohWerkst for publication, 2010.

[9] S. Aicher, G. Dill-Langer, and T. Ringger, "Non-destructive detection of longitudinal cracks in glulam beams," Otto-Graf-Journal, vol. 13, pp. 165-181, 2002.

[10] G. Dill-Langer, S. Aicher, and W. Bernauer, "Reflection measurements at timber glue-lines by means of ultrasound shear waves," Otto-GrafJournal, vol. 16, pp. 273-283, 2005.

[11] S. J. Sanabria, C. Mueller, J. Neuenschwander, P. Niemz, and U. Sennhauser, "Air-Coupled Ultrasound as an Accurate and Reproducible Method for Bonding Assessment of Glued Timber," Wood Science and Technology, vol. doi: 10.1007/s00226-010-0357-z, 2010.

[12] E. Blomme, D. Bulcaen, T. Cool, F. Declercq, and P. Lust, "Air-coupled ultrasonic assessment of wood veneer," Ultrasonics, vol. 50, pp. 180187, 2010.

[13] R. Y. Vun, K. Hoover, J. Janowiak, and M. Bhardwaj, "Calibration of non-contact ultrasound as an online sensor for wood characterization: Effects of temperature, moisture, and scanning direction," Applied Physics a-Materials Science \& Processing, vol. 90, pp. 191-196, 2008.

[14] H. Maurer, S. I. Schubert, F. Bachle, S. Clauss, D. Gsell, J. Dual, and P. Niemz, "A simple anisotropy correction procedure for acoustic wood tomography," Holzforschung, vol. 60, pp. 567-573, Aug 2006.

[15] H. Berndt, A. P. Schniewind, and G. C. Johnson, "Ultrasonic energy propagation through wood: where, when, how much," in 12th International Symposium on Nondestructive Testing of Wood, Sopron, Hungary, 2000, pp. 57-65.

[16] M. J. P. Musgrave, Crystal acoustics. San Francisco: Holden-Day, 2003

[17] V. Bucur, Acoustics of wood, 2nd ed. Berlin: Springer, 2006.

[18] H. Hoerig, "Anwendung der Elastizitätstheorie anisotroper Körper auf Messungen an Holz," Archive of Applied Mechanics, vol. 6, pp. 8-14, 1935.

[19] S. J. Sanabria, J. Neuenschwander, P. Niemz, and U. Sennhauser, "Structural health monitoring of glued laminated timber with a novel aircoupled ultrasound method," in 11th World Conference on Timber Engineering, Riva del Garda, Trentino, Italy, 2010.

[20] E. H. Saenger, N. Gold, and S. A. Shapiro, "Modeling the propagation of elastic waves using a modified finite-difference grid," Wave Motion, vol. 31, pp. 77-92, Jan 2000.

[21] S. J. Sanabria, R. Furrer, J. Neuenschwander, P. Niemz, and U. Sennhauser, "Air-Coupled Ultrasound Wave Propagation in Glued Laminated Timber," Holzforschung, Submitted for publication, 2010.

Fig. 5 Wave propagation and saw cut defect imaging in seven layers glulam. 